\title{
X-ray magnetic circular dichroism discloses surface spins correlation in maghemite hollow nanoparticles
}

Valentina Bonanni, Martina Basini, Davide Peddis, Alessandro Lascialfari, Giorgio Rossi, and Piero Torelli

Citation: Appl. Phys. Lett. 112, 022404 (2018);

View online: https://doi.org/10.1063/1.5006153

View Table of Contents: http://aip.scitation.org/toc/apl/112/2

Published by the American Institute of Physics

\section{Articles you may be interested in}

Modulated spin orbit torque in a Pt/Co/Pt/YIG multilayer by nonequilibrium proximity effect

Applied Physics Letters 112, 022402 (2018); 10.1063/1.5006115

Interface-induced perpendicular magnetic anisotropy of Co nanoparticles on single-layer h-BN/Pt(111)

Applied Physics Letters 112, 022407 (2018); 10.1063/1.5010836

Influence of $\mathrm{MgO}$ barrier quality on spin-transfer torque in magnetic tunnel junctions

Applied Physics Letters 112, 022406 (2018); 10.1063/1.5005893

Characterization of spin-transfer-torque effect induced magnetization dynamics driven by short current pulses Applied Physics Letters 112, 022401 (2018); 10.1063/1.5011721

Localized surface plasmon resonance enhanced magneto-optical Kerr effect in $\mathrm{Ni}_{80} \mathrm{Fe}_{20}$ thin films coated with Au nanorods

Applied Physics Letters 112, 022403 (2018); 10.1063/1.5011980

Selective control of vortex polarities by microwave field in two robustly synchronized spin-torque nano-oscillators Applied Physics Letters 112, 022405 (2018); 10.1063/1.5012768

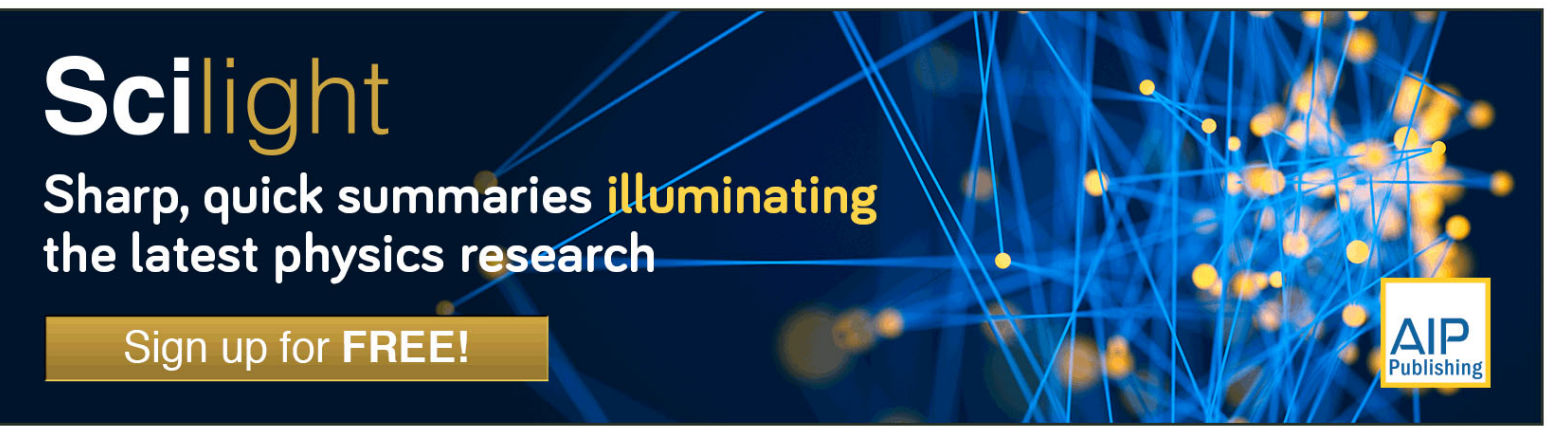




\title{
X-ray magnetic circular dichroism discloses surface spins correlation in maghemite hollow nanoparticles
}

\author{
Valentina Bonanni, ${ }^{1,2}$ Martina Basini, ${ }^{1}$ Davide Peddis, ${ }^{3}$ Alessandro Lascialfari, ${ }^{1}$ \\ Giorgio Rossi, ${ }^{1,2}$ and Piero Torelli ${ }^{2}$ \\ ${ }^{1}$ Department of Physics, Università degli Studi di Milano, Via Celoria 16, I-20133 Milano, Italy \\ ${ }^{2}$ IOM-CNR, S.S. $14 \mathrm{~km}$ 163.5, Basovizza, I-34149 Trieste, Italy \\ ${ }^{3}$ ISM-CNR, Via Salaria, Km 29.300,00015 Monterotondo Scalo (RM), Italy
}

(Received 22 September 2017; accepted 18 December 2017; published online 8 January 2018)

\begin{abstract}
The spin-spin correlations in hollow (H) and full (F) maghemite nanoparticles (NPs) have been studied by X-ray magnetic circular dichroism (XMCD). An unexpected XMCD signal was detected and analyzed under the application of a small field $\left(\mu_{0} \mathrm{H}=160 \mathrm{Oe}\right)$ and at remanence for both $\mathrm{F}$ and H NPs. Clear differences in the magnitude and in the lineshape of the XMCD spectra between $\mathrm{F}$ and H NPs emerged. By comparing XMCD measurements performed with a variable degree of surface sensitivity, we were able to address the specific role played by the surface spins in the magnetism of the NPs. Published by AIP Publishing. https://doi.org/10.1063/1.5006153
\end{abstract}

Spinel iron oxide magnetic nanoparticles (MNPs) have received increasing attention due to their expanding application fields from catalysis ${ }^{1}$ to biomedicine as magnetic hyperthermia therapy $y^{2,3}$ or enhanced magnetic resonance imaging. ${ }^{4}$ The understanding of the spin state and dynamics in such structures is a prerequisite to enable the manipulation of their magnetic functionalities.

The magnetism of MNPs is dominated by a single domain behavior for which both the temperature and the time-scale of the measurements control the magnetic response. When a static magnetic field is applied, a net remanent magnetization can be detected if the temperature is lower than a critical value $T_{B}$ (blocking temperature) above which the superparamagnetic regime occurs. For $\mathrm{T}<\mathrm{T}_{\mathrm{B}}$, the MNP spins are blocked and, according to the Néel model, the $T_{B}$ depends on the volume of the NPs. It is thus crucial that the synthesized NPs have sufficiently narrow volume distribution in order to contribute similarly to the collective magnetic behavior.

A crucial issue not taken into account in this oversimplified model is the magnetic behavior of the spins located at the MNP surface. In particular the magnetic dynamics, reflected in the blocking temperature, can be dramatically different for the surface spins with respect to the core ones.

Surface spin dynamics depends on the uncompensated atomic structure typical of the surface geometry and the changes of the oxidation state due to adsorbates and contaminants. Remarkably, element sensitive techniques like x-ray absorption spectroscopy (XAS) and x-ray magnetic circular dichroism (XMCD) provide information related to both phenomena.

Among the numerous MNP geometries, the hollow $(\mathrm{H})$ spheres are convenient nanosystems to explore the spin behavior at the surface due to their enhanced surface to volume ratio. ${ }^{5-8}$ Moreover, they are promising for applications as drug delivery vehicles ${ }^{9}$ thanks to their envisaged possibility to encapsulate the drug inside. Despite the growing interest on this class of NPs, demonstrated by the large amount of recently published works, there are no studies exploiting XAS and XMCD measurements. The comparison with the reduced surface to bulk ratio in full (F) NPs allows us to analyze the XMCD data, aiming to extract information on the surface spin magnetic behavior.

$\mathrm{XMCD}$ at the $\mathrm{L}_{2,3}$ edges of transition elements is a spectroscopy technique widely employed in the study of ferriand ferromagnetic oxides. Reference XAS/XMCD data of iron ferrites are usually used as a fingerprint of the oxidation states of this class of materials. ${ }^{10,11}$ Moreover, the elementspecific spin and orbital magnetic moments can be determined from experiments ${ }^{12}$ by applying robust sum rules. ${ }^{13,14}$ Typically, the absorption spectra are recorded at low temperature (where the NPs are magnetically blocked) while applying an external magnetic field of a few Tesla in order to magnetically saturate the system.

In this work, we have conceived a low-field (160 Oe) XMCD measurement procedure performing the spectra acquisition both at remanence and applying the external magnetic field in order to discern different magnetic components which may not be detectable applying a high magnetic field. It is worth highlighting that a significant XMCD signal has been measured and analyzed in maghemite NPs under the application of such a low magnetic field.

Three different specimens have been studied: two $\mathrm{H}$ and one F NPs. F NPs have been synthesized using the protocol proposed by Sun et al. ${ }^{15}$ The H NPs have been synthesized following a well-established procedure based on the Kirkendall effect. ${ }^{16} \mathrm{Fe}(\mathrm{Co}) 5$ is decomposed in air free conditions at around $220{ }^{\circ} \mathrm{C}$ in organic solvents. ${ }^{17}$ The resulting iron based NPs are oxidized in solution by means of oleic acid. Due to the self-diffusion for iron faster than oxygen ions, the iron oxide NPs turn into $\mathrm{H}$ iron oxide nanostructures.

TEM analysis confirms the presence of $\mathrm{H}$ and $\mathrm{F}$ spherical NPs with external diameter log-normal distributed. Mean values of the external diameter are 13 (2) $\mathrm{nm}$ and 7.0 (7) nm for $\mathrm{H}$ NPs (samples will be labeled $\mathrm{H} 1$ and $\mathrm{H} 2$, respectively) and 5.0 (5) nm for F NPs (F2). The inset of Fig. 1 shows the STEM of the sample $\mathrm{H} 2$.

An accurate magnetic characterization performed on the three samples has revealed a negligible interparticle 


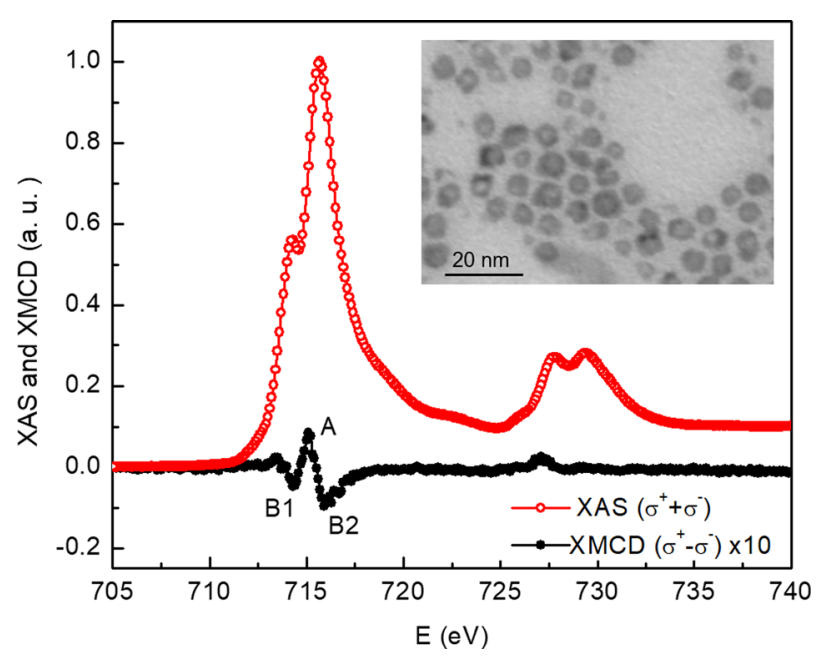

FIG. 1. XAS (open circles) and XMCD (solid circles) spectra at $\mathrm{Fe} \mathrm{L}_{2,3}$ edges of the sample $\mathrm{H} 2$ at $70 \mathrm{~K}$ with a magnetic field of $160 \mathrm{Oe}$. The positive peak at $715 \mathrm{eV}$ is indicated as $\mathrm{A}$, and the negative peaks at 714 and $716 \mathrm{eV}$ are indicated as B1 and B2, respectively. The inset shows the STEM of sample $\mathrm{H} 2$.

interaction and a complex polycrystalline structure in the $\mathrm{H}$ NPs with a consequence of high effective magnetic anisotropy. ${ }^{18}$

The specimens are prepared for the spectroscopic characterization dropping the NP solution on a Si substrate. The XAS and XMCD measurements are performed at the APEHigh Energy beamline of IOM-CNR (www.Trieste.NFFA.eu) at the Elettra-Sincrotrone Trieste facility ${ }^{19}$ in the totalelectron yield (TEY) detection mode at $70 \mathrm{~K}$ and an X-ray beam incidence of $55^{\circ}$ from the sample surface. The XMCD spectra were measured at the $\mathrm{Fe}_{2,3}$ absorption edges following two different procedures. In procedure \#1, while keeping the external magnetic field fixed at 160 Oe (parallel to the substrate surface), circularly polarized X-ray absorption is measured reversing the light helicity: clockwise $\left(\sigma^{+}\right)$and anti-clockwise $\left(\sigma^{-}\right)$. The unpolarized XAS is defined as the sum of $\sigma^{+}$and $\sigma^{-}$, whereas the XMCD is defined as the difference $\left(\sigma^{+}-\sigma^{-}\right)$. XAS and XMCD curves have been then normalized to the sum of the two circularly polarized spectra. In procedure \#2, the XMCD spectrum has been measured at a constant helicity of the X-ray but switching the applied magnetic field (switching time $\approx 1 \mathrm{~s}$ ) between plus and minus 160 Oe for each energy point of the XAS spectrum and measuring at remanence.

In both the procedures, the spectra are normalized to the incident beam intensity using the electron yield from a grid placed after the last optical element of the beamline.

Figure 1 shows the XAS and XMCD spectra of the sample $\mathrm{H} 2$, collected through procedure \#1 and representative of all the three specimens. The XAS line shape is characteristic of the $\gamma-\mathrm{Fe}_{2} \mathrm{O}_{3}$ phase ${ }^{20-22}$ where the trivalent $\mathrm{Fe}$ ions distributed between tetrahedral and octahedral sites are identified by a low energy shoulder of the main peak of the $\mathrm{Fe} \mathrm{L}_{3}$ edge and in a double peak structure at the $\mathrm{Fe} \mathrm{L}_{2}$ edge. The antiferromagnetic coupling of the $\mathrm{Fe}^{3+}$ ions in tetrahedral and octahedral sites is reflected in a XMCD spectrum with a typical three-peak shape at the $\mathrm{Fe} \mathrm{L}_{3}$ edge. The positive peak at $715 \mathrm{eV}$ (marked as A) is mostly ascribable to the $\mathrm{Fe}^{3+}$ ions in tetrahedral sites, whereas the two negative peaks at 714 (marked as B1) and $716 \mathrm{eV}$ (marked as B2) originate from $\mathrm{Fe}^{3+}$ ions in octahedral sites. ${ }^{23}$ The corresponding features at the $\mathrm{Fe} \mathrm{L}_{2}$ edge are difficult to observe due to the overall small intensity, and thus, we will concentrate on the analysis of the XAS/XMCD spectra in the $\mathrm{Fe}_{3}$ edge henceforth.

By comparing the $\mathrm{Fe}_{3}$ XMCD spectra (procedure \#1) in Fig. 2(a) for all the three samples, it emerges that the intensity of the signal for the F NP system (F2) is about twice as large as the one of the H NPs (H1 and H2). The larger XMCD signal in the F NPs indicates a higher magnetic moment as compared to the H NPs. These results are consistent with the SQUID magnetometer measurements [Fig. 2(b)], where the F NPs show the highest magnetic moment among the three samples.

The overall lineshape is similar for the three samples, revealing a stable state of oxidation of the specimens' batch without significant uncontrolled oxidation processes taking place. ${ }^{24}$ However, a closer inspection reveals a deviation of the $\mathrm{H} 2$ spectrum from the other two samples ( $\mathrm{H} 1$ and $\mathrm{F} 2$ ), in particular in the energy region between $716.5 \mathrm{eV}$ and $720 \mathrm{eV}$. It is not possible to analyze the origin of this difference simply based on the convolution of the XMCD signal from the $\mathrm{Fe}^{3+}$ ions in octahedral and tetrahedral sites. However, it is possible to relate this different spectral shape to a variation of the ratio of the number of tetrahedral and octahedral sites for the $\mathrm{H} 2$ system compared to $\mathrm{H} 1$ and F2 samples. In order to verify this hypothesis and clarify the observed differences, additional investigation is required.

The same samples were analyzed also with procedure \#2 (Fig. 3): the signal is significantly smaller than the one
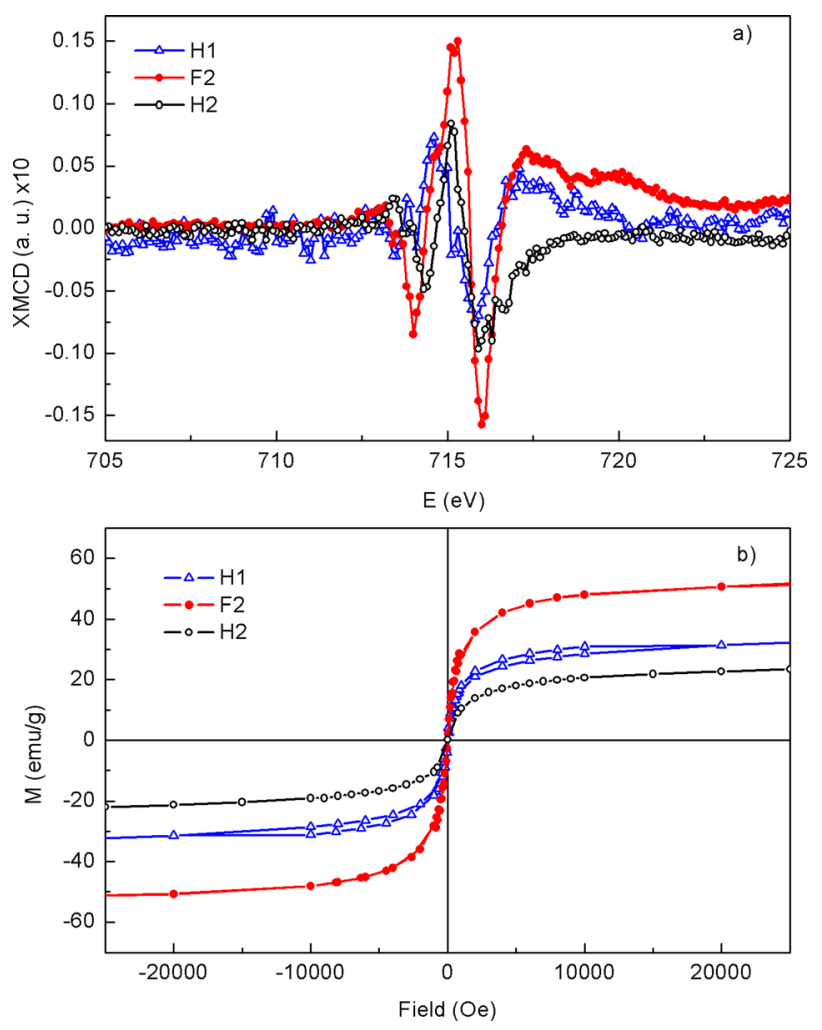

FIG. 2. XMCD spectra at the Fe $\mathrm{L}_{3}$ edge of samples $\mathrm{H} 1$ (open triangles), $\mathrm{H} 2$ (open circles), and F2 (full circles) following procedure \#1 [panel (a)]; hysteresis loops of samples H1 (open triangles), H2 (open circles), and F2 (full circles) at $70 \mathrm{~K}$ [panel (b)]. 


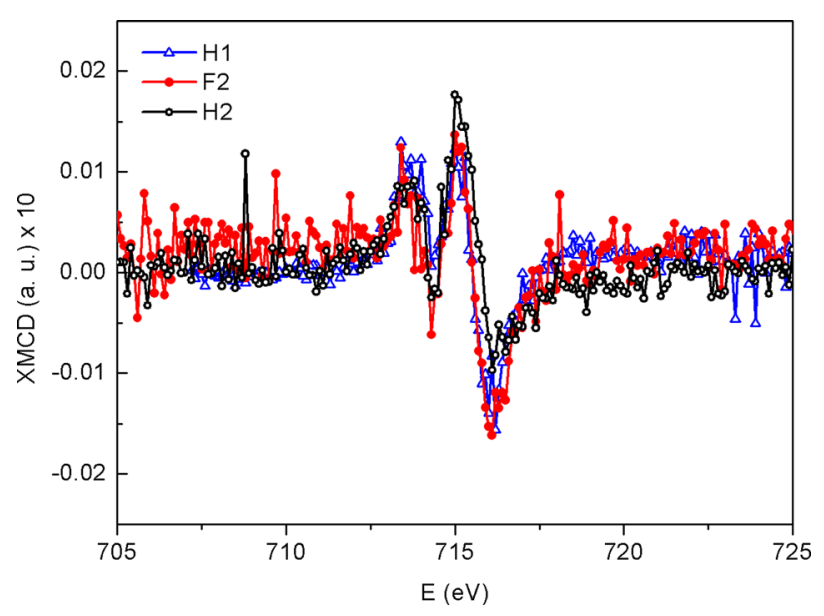

FIG. 3. XMCD spectra at the $\mathrm{Fe}_{3}$ edge of samples $\mathrm{H} 1$ (open triangles), $\mathrm{H} 2$ (open circles), and F2 (full circles) following procedure \#2.

measured with the applied field consistently with a reduced magnetic correlation at remanence. Remarkably, the XMCD signal at remanence is small but measureable for both $\mathrm{F}$ and $\mathrm{H} \gamma-\mathrm{Fe}_{2} \mathrm{O}_{3}$ NPs. Unlike procedure \#1, the XMCD recorded with procedure \#2 does not show differences between $\mathrm{H}$ and F NPs. Thus, while the response to the applied field changes with the shape of the NPs, the remanence seems to be unaffected. It is also worth noting that the shape of the XMCD measured at remanence (procedure \#2) is different from the one in the presence of the applied magnetic field (procedure $\# 1$ ). This difference indicates that two independent magnetic contributions are superimposed: the XMCD features measured at remanence may be attributed to mono-domain NPs in the blocked phase, while the additional XMCD features that add to the spectra when the applied field is on may be attributed to an additional paramagnetic contribution. The two contributions could arise from different environments in the NPs, indicating the coexistence of two magnetically active sites. This explanation could be in agreement with the presence of two main NP populations where the bigger NPs are blocked at $70 \mathrm{~K}$ and the smaller ones are in the superparamagnetic regime.

In order to investigate if the surface spins play a distinctive role in the F and H NPs, we have also performed partial electron yield (PEY) XAS/XMCD measurements (applying a positive potential of $200 \mathrm{~V}$ to the sample).

The PEY method is based on an electrostatic selection of the range of kinetic energies of the detected electrons such as to limit the retrieved signal from the uppermost layers of the material. PEY data are therefore more surface sensitive than TEY data. ${ }^{25}$ According to the literature, the TEY probing depth is about $3.5 \mathrm{~nm}$ in $\mathrm{Fe}_{2} \mathrm{O}_{3},{ }^{26}$ whereas the PEY attenuation length can be estimated to be around $2 \mathrm{~nm}^{27}$

Rescaling the spectra to the A peak, a reduction in the relative intensity of the B2 peak is observed in the PEY mode for one of the H NP systems (H1) compared to the F NP one (F2) [Fig. 4(a)]. The same intensity reduction is observed if we compare the rescaled XMCD spectra collected with TEY and PEY modes for H1 [Fig. 4(b)]. Unlike sample F2, where the TEY and PEY spectra overlap at all the three $\mathrm{Fe}_{3}$ edge peaks [Fig. 4(c)], in the $\mathrm{H}$ system $\mathrm{H} 1$, still the negative peak B2 shows a reduced intensity when
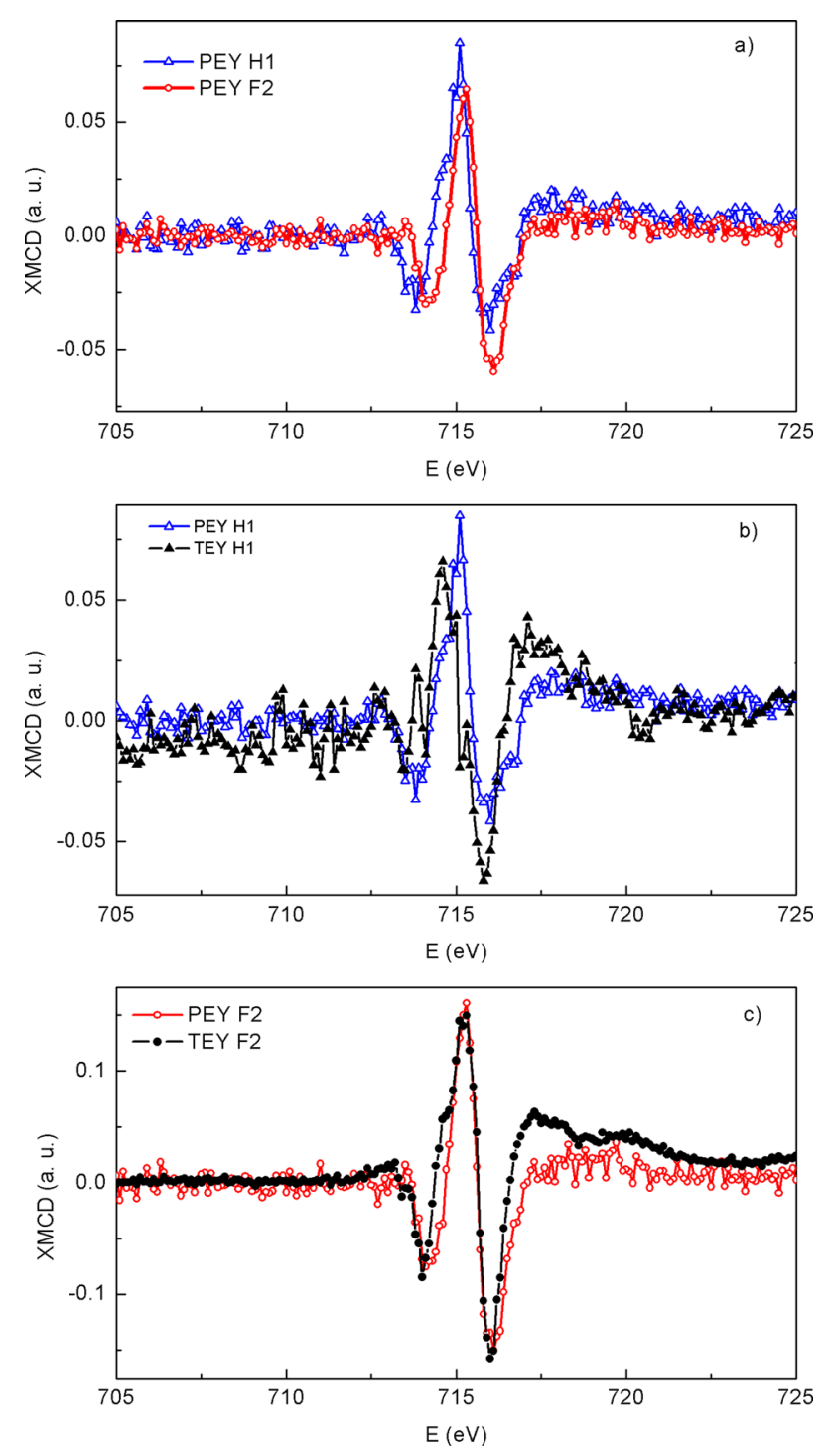

FIG. 4. XMCD recorded in the PEY mode for samples H1 (open triangles) and F2 (open circles) at the Fe L3 edge [panel (a)]; XMCD recorded in PEY (open triangles) and TEY (solid triangles) for sample H1 [panel (b)]; XMCD recorded in PEY (open circles) and TEY (solid circles) for sample F2 [panel (c)]. The spectra are rescaled according to the overlapping of the positive peak A.

measured in the PEY mode, i.e., when the sensitivity to the external surface of the NP shell is the highest.

We attribute the reduction of the $\mathrm{B} 2$ peak intensity to a reduced correlation among the spins of the external surface of the NPs, consistently to what is reported in the literature. ${ }^{21}$ A previous study of maghemite NPs by means of a combination of XAS/XMCD and Mössbauer techniques ${ }^{21}$ has evidenced a greater disorder of $\mathrm{Fe}^{3+}$ spins in octahedral sites than for $\mathrm{Fe}^{3+}$ spins in tetrahedral sites under the application of a low magnetic field.

It is important to highlight that also the negative peak B1 should be affected by an intensity reduction, similar to the B2 peak, but we are not able to experimentally observe this variation. The reason may be related to the overall small intensity of our signal due to the small magnetic field applied which restricts the discussion to the more intense B2 peak.

Our PEY XMCD gives evidence of a weaker exchange interaction among surface spins in the H NPs as compared to 
the $\mathrm{F}$ ones, even if the overall electronic configuration is reflected in identical XAS spectra, within the experimental sensitivity. It could be found that the same reduction of the magnetic correlation among the surface spins occur also in the F NPs but only increasing the surface to volume ratio, through the $\mathrm{H}$ geometry, this effect becomes detectable. Our results are consistent with the accepted magnetic behavior scenario $^{5,28,29}$ of the H NPs, which is characterized by a decrease in the magnetization of the system and an increase in the effective anisotropy and the exchange bias effect.

However, the low magnitude of the applied magnetic field does not allow addressing the saturation magnetization or anisotropy energy, but it is appropriate to disentangle the coexistence of a blocked superparamagnetism at $70 \mathrm{~K}$ and an additional paramagnetic contribution, probably due to a coexistence of a bigger (spin-blocked at $70 \mathrm{~K}$ ) and a smaller (superparamagnetic at $70 \mathrm{~K}$ ) MNP population.

In summary, the analysis of the XAS spectra shows that the oxidation state in the maghemite NPs is identical in both the $\mathrm{F}$ and $\mathrm{H}$ structures. However, the XMCD spectra at a low magnetic field reveal a reduced magnetization in the $\mathrm{H}$ NPs compared to the F ones. The comparison between the XMCD spectra measured at remanence and in the presence of an applied (low) magnetic field of 160 Oe reveals the coexistence of two NP populations with different sizes and thus with a different magnetic response (spin blocked and superparamagnetic regimes). Surface sensitive data indicate that a reduced magnetic correlation among the spins occurs at the external surface of the H NPs, different from the F NPs. We show that XMCD can be measured in iron oxide NPs even when applying a low magnetic field (160 Oe). Combining the peculiar enhancement of the surface to volume ratio given by the $\mathrm{H}$ geometry with the versatility of the XMCD technique in probing a variable degree of surface sensitivity, we conceived an experiment which allows detecting the surface spin magnetic behavior.

This work was performed in the framework of the nanoscience foundry and fine analysis facility (NFFA-MIUR Italy Progetti Internazionali). V. B., M.B., and A.L. thank the INSTM-Regione Lombardia Project "MOTORSPORT" and the EU-COST RADIOMAG No. TD1402.

\footnotetext{
${ }^{1}$ M. Hermanek, R. Zboril, I. Medrik, J. Pechousek, and C. Gregor, J. Am. Chem. Soc. 129, 10929 (2007).

${ }^{2}$ N. Tran and T. J. Webster, J. Mater. Chem. 20, 8760 (2010).

${ }^{3}$ J.-P. Fortin, C. Wilhelm, J. Servais, C. Ménager, J.-C. Bacri, and F. Gazeau, J. Am. Chem. Soc. 129, 2628 (2007).

${ }^{4}$ J. Kim, Y. Piao, and T. Hyeon, Chem. Soc. Rev. 38, 372 (2009).
}

${ }^{5}$ H. Khurshid, W. Li, M.-H. Phan, P. Mukherjee, G. C. Hadjipanayis, and H. Srikanth, Appl. Phys. Lett. 101, 022403 (2012).

${ }^{6}$ E. V. Shevchenko, M. I. Bodnarchuk, M. V. Kovalenko, D. V. Talapin, R. K. Smith, S. Aloni, W. Heiss, and A. P. Alivisatos, Adv. Mater. 20, 4323 (2008).

${ }^{7}$ D. Goll, A. E. Berkowitz, and H. N. Bertram, Phys. Rev. B 70, 184432 (2004).

${ }^{8}$ G. H. Jaffari, A. Ceylan, H. P. Bui, T. P. Beebe, Jr., S. Ozcan, and S. I. Shah, J. Phys. Condens. Matter 24, 336004 (2012).

${ }^{9}$ R. Xing, A. A. Bhirde, S. Wang, X. Sun, G. Liu, Y. Hou, and X. Chen, Nano Res. 6, 1 (2013).

${ }^{10}$ P. Kuiper, B. Searle, L.-C. Duda, R. Wolf, and P. van der Zaag, J. Electron Spectrosc. Relat. Phenom. 86, 107 (1997).

${ }^{11}$ E. Lee, D. H. Kim, J. Hwang, K. Lee, S. Yoon, B. J. Suh, K. Hyun Kim, J.-Y. Kim, Z. H. Jang, B. Kim, B. I. Min, and J.-S. Kang, Appl. Phys. Lett. 102, 133703 (2013).

${ }^{12}$ J. A. Moyer, C. A. F. Vaz, D. A. Arena, D. Kumah, E. Negusse, and V. E. Henrich, Phys. Rev. B 84, 054447 (2011).

${ }^{13}$ B. T. Thole, P. Carra, F. Sette, and G. van der Laan, Phys. Rev. Lett. 68 , 1943 (1992).

${ }^{14}$ P. Carra, B. T. Thole, M. Altarelli, and X. Wang, Phys. Rev. Lett. 70, 694 (1993).

${ }^{15}$ S. Sun, H. Zeng, D. B. Robinson, S. Raoux, P. M. Rice, S. X. Wang, and G. Li, J. Am. Chem. Soc. 126, 273 (2004).

${ }^{16}$ H. J. Fan, U. Gösele, and M. Zacharias, Small 3, 1660 (2007).

${ }^{17}$ P. A. Kumar, G. Singh, W. R. Glomm, D. Peddis, E. Wahlström, and R. Mathieu, Mater. Res. Express 1, 036103 (2014).

${ }^{18} \mathrm{M}$. Basini, "Effect of the hollow topology on the spin dynamics in iron oxide magnetic nanoparticles," Doctoral thesis, Università degli Studi di Milano, 2017.

${ }^{19}$ G. Panaccione, I. Vobornik, J. Fujii, D. Krizmancic, E. Annese, L. Giovanelli, F. Maccherozzi, F. Salvador, A. De Luisa, D. Benedetti, A. Gruden, P. Bertoch, F. Polack, D. Cocco, G. Sostero, B. Diviacco, M. Hochstrasser, U. Maier, D. Pescia, C. H. Back, T. Greber, J. Osterwalder, M. Galaktionov, M. Sancrotti, and G. Rossi, Rev. Sci. Instrum. 80, 043105 (2009).

${ }^{20}$ E. Pellegrin, M. Hagelstein, S. Doyle, H. O. Moser, J. Fuchs, D. Vollath, S. Schuppler, M. A. James, S. S. Saxena, L. Niesen, O. Rogojanu, G. A. Sawatzky, C. Ferrero, M. Borowski, O. Tjernberg, and N. B. Brookes, Phys. Status Solidi B 215, 797 (1999).

${ }^{21}$ S. Brice-Profeta, M.-A. Arrio, E. Tronc, N. Menguy, I. Letard, C. Cartier dit Moulin, M. Noguès, C. Chanéac, J.-P. Jolivet, and P. Sainctavit, J. Magn. Magn. Mater. 288, 354 (2005).

${ }^{22}$ Y. Prado, N. Daffé, A. Michel, T. Georgelin, N. Yaacoub, J.-M. Grenèche, F. Choueikani, E. Otero, P. Ohresser, M.-A. Arrio, C. Cartier-dit-Moulin, P. Sainctavit, B. Fleury, V. Dupuis, L. Lisnard, and J. Fresnais, Nat. Commun. 6, 10139 (2015).

${ }^{23}$ V. N. Antonov, B. N. Harmon, and A. N. Yaresko, Phys. Rev. B 67, 024417 (2003).

${ }^{24}$ C. Graf, C. Goroncy, P. Stumpf, E. Weschke, C. Boeglin, H. Ronneburg, and E. Rühl, J. Phys. Chem. C 119, 19404 (2015).

${ }^{25}$ J. Stöhr, C. Noguera, and T. Kendelewicz, Phys. Rev. B 30, 5571 (1984).

${ }^{26}$ S. Gota, M. Gautier-Soyer, and M. Sacchi, Phys. Rev. B 62, 4187 (2000).

${ }^{27} \mathrm{C}$. J. Powell and A. Jablonski, NIST Electron Effective-Attenuation-Length Database - Version 1.3 (National Institute of Standards and Technology, Gaithersburg, MD, 2011).

${ }^{28}$ A. Cabot, A. P. Alivisatos, V. F. Puntes, L. Balcells, Ò. Iglesias, and A. Labarta, Phys. Rev. B 79, 094419 (2009).

${ }^{29}$ Z. Nemati, H. Khurshid, J. Alonso, M. H. Phan, P. Mukherjee, and H. Srikanth, Nanotechnology 26, 405705 (2015). 